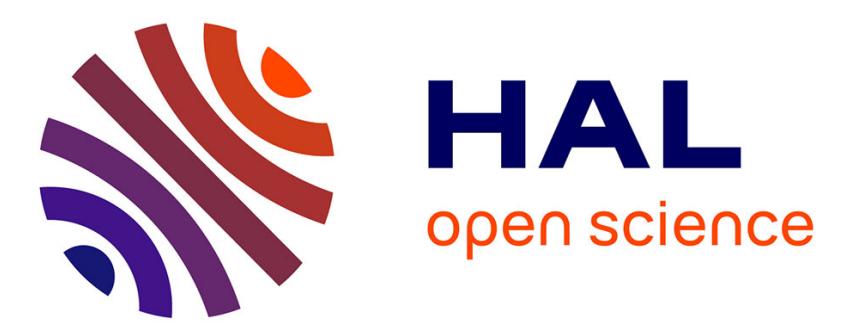

\title{
CMOS 80 K-300 K SPICE Parameter for IRFPA Readout Circuit Design
}

\author{
W.-L. Lu, Z.-W. Hwang, L. Lu
}

\section{To cite this version:}

W.-L. Lu, Z.-W. Hwang, L. Lu. CMOS 80 K-300 K SPICE Parameter for IRFPA Readout Circuit Design. Journal de Physique IV Proceedings, 1996, 06 (C3), pp.C3-199-C3-206. 10.1051/jp4:1996330 . jpa-00254248

\section{HAL Id: jpa-00254248 https://hal.science/jpa-00254248}

Submitted on 1 Jan 1996

HAL is a multi-disciplinary open access archive for the deposit and dissemination of scientific research documents, whether they are published or not. The documents may come from teaching and research institutions in France or abroad, or from public or private research centers.
L'archive ouverte pluridisciplinaire HAL, est destinée au dépôt et à la diffusion de documents scientifiques de niveau recherche, publiés ou non, émanant des établissements d'enseignement et de recherche français ou étrangers, des laboratoires publics ou privés. 


\title{
CMOS 80 K-300 K SPICE Parameter for IRFPA Readout Circuit Design
}

\author{
W.-L. Lu, Z.-W. Hwang and L.S. Lu* \\ Dept. of Physics and Chemistry, Chinese Naval Academy, Tso-Ying, Kaohsiung, Taiwan, R.O.C. \\ * Dept. of Applied Physics, Chung Cheng Institute of Technology, Tshsi, Taoyuan, Taiwan, R.O.C.
}

\begin{abstract}
This work presents the low temperature characteristics of commercial complementary-mentaloxide-serniconductor devices, which may be used in the readout electronics of the infrared focal plane array detector. Measurements are performed with the temperature calibration of the device under test. Two most important temperature dependent parameters of the drain current characteristics of the device, the threshold voltage and the carrier mobility are studied. Carrier freeze out effect is considered in the threshold voltage calculation. Two kinds of empirical carrier mobility model are studied, and better model is included in the SPICE mobility model. A revised SPICE MOS level 3 parameter extraction scheme is proposed to extract new mobility model parameters.
\end{abstract}

\section{Introduction}

Large detector arrays used for thermal imaging may consists of $10^{5}$ or more detectors, making it impractical to use discrete devices for signal processing (include both pre-amplifier and multiplexer ). In this regard, silicon VLSI processes are used, either on the same chip with the detector or on a separate chip in a hybrid configuration, to fabricate this readout electronics[1]. In a high performance liquid nitrogen cooled IRFPA (infrared focal plane array), after the detectors convert the detected photons into electrical charge, the resulting signal is injected into the readout circuits for multiplexing[1]. In the commonly used hybrid configuration, this input circuit must be designed to work at liquid nitrogen temperature and with specialized input circuits that meet the high performance specification and yet have very little available area for complex circuitry,

As the injection efficiency of the input circuit depends mainly on the transconductance of the MOSFET [1], it is required that a more accurate low temperature SPICE parameter being extracted. According to the drain current equation of the MOSFET device (in linear region) [2]

$$
I_{D S}=\mu_{e f f} C_{o x} \frac{W}{L}\left(V_{G S}-V_{T H}-\frac{1}{2} V_{D S}\right) V_{D S}
$$

where $I_{D S}$ and $V_{D S}$ represents drain current and drain bias, $V_{G S}$ and $V_{T H}$ represents gate bias and threshold voltage, $\mu_{\text {eff }}$ is the carrier effective mobility, and $W$ and $L$ represents channel width and channel length respectively. There are two most important temperature dependent parameters in equation (1), namely, the threshold voltage $\left(\mathrm{V}_{\mathrm{TH}}\right)$ and the carrier effective mobility $\left(\mu_{\mathrm{eff}}\right)$. The purpose of this work is to present a more accurate SPICE model for a wider temperature range, by suitably adjust the mobility model and the consideration of the freeze out effect on the threshold voltage.

As SPICE MOS level 3 model (with only $\sim 12$ fitting parameters) can be used to simulate device characteristics down to $\sim 2 \mu \mathrm{m}$ range with good results (in compare with $\sim 39$ fitting parameters of the BSIM3 model for simulation of device size down to $\sim 0.2 \mu \mathrm{m}$ range), the SPICE MOS level 3 model is used 
in our study to simplify the parameter extraction scheme but without the expenses of the validity of the temperature dependence of threshold voltage and carrier mobility model we choose in our study.

\section{Experimental Procedure}

The devices used in this study are fabricated by local commercial foundry, with devices of W/L=50 $\mu \mathrm{m}$ $150 \mu \mathrm{m}, 50 \mu \mathrm{m} / 1.2 \mu \mathrm{m}$, and $2 \mu \mathrm{m} / 50 \mu \mathrm{m}$. The devices under test (DUTs) are packaged with ceramic chip carriers and placed in a vacuum environment. A liquid nitrogen cooling system with temperature controller is used to provide the desired testing temperature. To ensure the accuracy of the low temperature measurement results, we have carefully calibrated the temperature of the DUT by attaching a calibrated silicon diode temperature sensor directly on top of the DUT. The calibration of the silicon diode temperature sensor is based on the International Temperature Scale of 1990 (ITS-90) and is traceable to the NIST standard. During the measurement, the settings of the temperature controller are based on the calibrated DUT temperature table. This ensures the DUT's temperature is within about $\pm 3 \mathrm{~K}$ of the desired setting in the $80 \mathrm{~K} \sim 100 \mathrm{~K}$ range. At higher temperatures the error si within $\pm 1 \mathrm{~K}$.

The transconductance change (TC) [3] method is used to extract device threshold voltage at various temperatures. TC method, which utilizes the gate voltage at which the second derivative of the drain current versus gate voltage curve is a peak as the threshold voltage, has been proven to get a more accurate threshold voltage for various channel implant profiles, and is relatively insensitive to interface states, normal field mobility degradation and device series resistance[4].

Carrier mobility is extracted from drain current $I_{D S}$ versus drain bias $\left(V_{D S}\right)$ curve at low drain bias $\left(V_{D S}\right.$ $=50 \mathrm{mV}$ ) condition. For large device structure $(\mathrm{W} / \mathrm{L}=50 \mathrm{~m} \mu / 50 \mu \mathrm{m})$, the channel conductance (defined as $\mathrm{g}_{\mathrm{d}}$ $\left.=\partial \mathrm{I}_{\mathrm{DS}} / \partial \mathrm{V}_{\mathrm{DS}}\right)$ is related to the carrier surface mobility as [5]

$$
\mu_{\mathrm{s}}=\frac{\partial \mathrm{I}_{\mathrm{DS}}}{\partial \mathrm{V}_{\mathrm{DS}}} \frac{\mathrm{L}}{\mathrm{W}} \frac{1}{\mathrm{Cox}_{\mathrm{ox}}\left(\mathrm{V}_{\mathrm{GS}} \mathrm{V}_{\mathrm{TH}}\right)}
$$

where $\mathrm{L}$ and $\mathrm{W}$ are effective channel length and width of the device, $\mathrm{C}_{\mathrm{ox}}$ is the gate oxide capacitance per unit area.

\section{Temperature dependence of threshold voltage}

Figure 1 (symbols) represents the extracted results of the temperature dependence of threshold voltage (by using the TC method to extract threshold voltage from the large area device). The equation for prediction of the freeze out effect on the threshold voltage can be found in numerous text books, but the actual calculation requires numerical techniques. Basically, in this work, we are following the methods suggest by Wang [6], and with minor correction on the coefficients of the equations. The threshold voltage of NMOSFET can be represented as [7]

$$
\mathrm{V}_{\mathrm{T} 0}=\phi_{\mathrm{ms}}+2 \phi_{\mathrm{F}}-\frac{\mathrm{Q}_{\text {tot }}}{\mathrm{C}_{\mathrm{ox}}}+\frac{\mathrm{Q}_{\mathrm{BO}}}{\mathrm{C}_{\mathrm{ox}}}
$$

where $\mathrm{V}_{\mathrm{TO}}$ represents the threshold voltage at zero substrate bias, $\phi_{\mathrm{ms}}=\phi_{\mathrm{F}}$ (poly gate) $-\phi_{\mathrm{F}}(\mathrm{Si}), \mathrm{q} \phi_{\mathrm{F}}=\mathrm{E}_{\mathrm{F}}$ $E_{i}, E_{F}$ and $E_{i}$ represents Fermi level and intrinsic level respectively, $\phi_{m s}$ represents device turn on condition, $Q_{\text {tot }}$ and $Q_{B O}$ represents fitting parameter and depletion region charge.

Secant method [8] is used in the calculation of the Fermi level from the charge neutrality equation [6], and the result is shown in figure 2 . After the Fermi level is calculated at certain temperature, $E_{1}, \phi_{F}, \phi_{\mathrm{ms}}$, and $\mathrm{Q}_{\mathrm{BO}}=\sqrt{4 \phi_{\mathrm{F}} \varepsilon_{\mathrm{si}} \mathrm{qN} \mathrm{N}_{\mathrm{sub}}}$ can be calculated subsequently. Equation 2 is then used to fit the measured data (in figure 1), whereas $\mathrm{Q}_{\text {tot }}$ (oxide charge + interface state charge) is used as the fitting parameter. The fitting curve is shown as solid line in figure 1 , and the results show that with reasonable quantity of $Q_{\text {tot }}$ 
$\left(\sim 4.9 \times 10^{11} \mathrm{~cm}^{-2}\right)$, quite good prediction of the temperature dependence of the threshold voltage can be achieved. Detail equations that used in our calculations can be found in Wang's work.

One point needs to probe further is that, the fitting result is based on the assumption that in the calculation of the bulk region charge $Q_{B O}=\sqrt{4 \phi_{\mathrm{F}} \varepsilon_{\mathrm{si}} \mathrm{q} \mathrm{N}_{\mathrm{sub}}}$, fixed substrate concentration (room temperature carrier concentration) is used in the value of $\mathrm{N}_{\mathrm{sub}}$. If freeze out effect is also considered in the calculation of $\mathrm{Q}_{\mathrm{BO}}$, the prediction result would show much error in compare with the experimental result. Furthermore, due to more complex channel doping process involved in fabricating PMOSFET $\left(\mathrm{P}^{+}\right.$counter doping implant is used to adjust threshold voltage shift by using $\mathrm{n}^{+}$poly as poly gate of PMOSFET), the freeze out effect may results on more complicated effects in the analysis of the temperature dependence of the threshold voltage. The theoretical prediction of temperature dependence of the threshold voltage for PMOSFET will not be discussed in this work. As for the future trend of using $\mathrm{P}^{+}$poly gate for the PMOSFET, the calculation in this work can be used to predict the temperature dependence of the threshold voltage.

\section{Temperature dependence of surface mobility}

Figure 3 (symbol) shows the measured result of surface mobility versus gate overdrive $\left(\mathrm{V}_{\mathrm{GS}}-\mathrm{V}_{\mathrm{TH}}\right)$ at various temperatures for NMOSFET. Theoretical prediction of carrier mobility from basic calculation of the scattering process is complex and tedious and is impractical to be used in the SPICE model. Recently, two empirical carrier mobility model have been proposed to represent the degradation of the carrier mobility under the influence of transverse field from the gate bias.

In light of the observed phenomena of linear relation between the value of $\left[\mathrm{I}_{\mathrm{DS}}^{2} /\left(\mathrm{d} \mathrm{I}_{\mathrm{DS}} / \mathrm{dV} \mathrm{VS}_{\mathrm{GS}}\right)\right]^{\frac{1}{n}}$ and gate bias (where $\mathrm{n}$ is a fitting value), and the bell shape function between surface mobility and gate overdrive (which also observed in our measured result in figure 3), Ghibaudo et al. proposed a simple empirical mobility model [9]

$$
\mu_{\mathrm{s}}=\frac{2 \mu_{\mathrm{m}}\left(\mathrm{V}_{\mathrm{GS}}-\mathrm{V}_{\mathrm{TH}}\right)}{1+\theta\left(\mathrm{V}_{\mathrm{GS}}-\mathrm{V}_{\mathrm{TH}}\right)^{2}}
$$

where $\mu_{s}$ represents surface mobility (for both electron and hole), $\mu_{m}$ and $\theta$ are the two fitting parameters. As another approach to develop a simple and yet efficient in computation time consumption, is by Tasch et al. They proposed an empirical electron mobility model to describe the electron and hole mobility degradation under gate bias [10]

$$
\mu_{\mathrm{s}}=\frac{\mu_{0}}{1+\theta_{1}\left(\mathrm{~V}_{\mathrm{GS}}-\mathrm{V}_{\mathrm{TH}}\right)^{0.25}+\theta_{2}\left(\mathrm{~V}_{\mathrm{GS}}-\mathrm{V}_{\mathrm{TH}}\right)}
$$

where $\mu_{0}, \theta_{1}$, and $\theta_{2}$ are all fitting parameters.

As for the comparison of the models presented by Ghibaudo et al. and Tasch et al., both empirical models are used to fit the experimental measured results. The widely used Levengerg-Marquardt (LM) nonlinear optimization method [8] is used in the fitting process, to extract the values of the fitting parameter in the models. The Ghibaudo's empirical model is first used to fit the experimental measured surface mobility (in figure 3 by using LM optimizer) for various temperatures. Temperature dependence of the two parameters thus extracted $\left(\mu_{m}\right.$ and $\theta$ ), show an exponential relationship with the temperature (symbols in figure 4). The solid lines in figure 4 represents prediction of the functions

and

$$
\mu_{\mathrm{m}}=7996.71 \mathrm{e}^{-7.32 \times 10^{-3} \mathrm{~T}}
$$

$$
\theta=0.402 \mathrm{e}^{-8.75 \times 10^{-4} \mathrm{~T}}
$$


(which is obtained by using linear regression to the data points larger than $80 \mathrm{~K}$ in the figure 4 semilog chart), and the solid line in figure 3 represents the simulation results of the Ghibaudo's model, by using the parameters in equations (6) and (7). As the results of Ghibaudo's model in figure 3 shows, the model can be used to predict electron mobility down to $\sim 40 \mathrm{~K}$, but with some error in the higher temperature $(>200 \mathrm{~K})$ and near low gate overdrive region.

Figure 5 shows the comparison between the measured (symbol) electron mobility and prediction of the Tasch's model (line). The same calculation steps as that reported in the above study of the Ghibaudo's model, the Tasch's empirical model is first used to fit the experimental measured surface mobility (in figure 5 , by using the LM optimizer) for various temperatures. The temperature dependence of the three parameters that being extracted $\left(\mu_{0}, \theta_{1}, \theta_{2}\right)$ also shows an exponential relationship with the temperature (symbols in figure 6). The solid lines in figure 6 represents prediction of the functions

and

$$
\begin{aligned}
& \mu_{0}=903.969 \mathrm{e}^{-4.1216 \times 10^{-3} \mathrm{~T}} \\
& \theta_{1}=-1.05689 \mathrm{e}^{-2.5789 \times 10^{-3} \mathrm{~T}}
\end{aligned}
$$

$$
\theta_{2}=0.1692 \mathrm{e}^{-2.2796 \times 10^{-3} \mathrm{~T}}
$$

(this is also obtained by using linear regression to the data points larger than $65 \mathrm{~K}$ in the figure 6 semilog chart), and the solid line in figure 5 shows the simulation results of the Tasch's model by using the parameters in equations (8), (9), and (10). From the results of Tasch's model shown in figure 5 , the model can be used to predict electron mobility at higher temperatures $>200 \mathrm{~K}$, and with a better prediction at low gate overdrive region.

As our application are focused at low temperature region and the consideration of computer time in SPICE simulation, Ghibauso's model with its more accurate prediction of electron mobility in low temperature region and with only two parameters to be calculated during simulation, seemed to be a better choice to be implement in the SPICE model for carrier mobility prediction. In the studies follows, we will use Ghibaudo's model to substitute the present mobility degradation model in SPICE MOS level 3.

\section{Mobility parameter extraction strategy}

As mentioned in section 1, our SPICE model for wide temperature range simulation is based on SPICE MOS level 3 model with the adjustment of the surface mobility model and the consideration of the freeze out effect on the threshold voltage. Since the theoretical prediction of the threshold voltage in section 3 is quite accurate, the prediction of threshold voltage in SPICE MOS level 3 is replaced here by using the method in section 3 .

The surface mobility model proposed in section 4 is used to substitute the original mobility model in SPICE MOS level 3. For large device $(\mathrm{W} / \mathrm{L} \sim 50 \mu \mathrm{m} / 50 \mu \mathrm{m})$ with low drain bias $\mathrm{V}_{\mathrm{DS}} \sim 50 \mathrm{mV}$, the drain current equation (level 3) can be written as

where

$$
I_{D S}=\beta_{\text {eff }} V_{D S}\left(V_{G S}-V_{\mathrm{TH}}-\frac{1}{2} V_{D S}\right)
$$

$$
\begin{aligned}
& \beta_{\text {eff }}=\mu_{\text {eff }} C_{\text {ox }} \frac{W_{\text {eff }}}{L_{\text {eff }}} \\
& \mu_{\text {eff }}=\frac{\mu_{\mathrm{s}}}{1+\frac{\mu_{s} v_{D S}}{v_{\max } \underline{L}_{\text {eff }}}}
\end{aligned}
$$

and

$$
\mu_{\mathrm{s}}=\frac{2 \mu_{\mathrm{m}}\left(\mathrm{V}_{\mathrm{GS}}-\mathrm{V}_{\mathrm{TH}}\right)}{1+\theta\left(\mathrm{V}_{\mathrm{GS}} \mathrm{V}_{\mathrm{TH}}\right)^{2}}
$$


where $\mu_{\text {eff }}, W_{\text {eff }}$, and $L_{\text {eff }}$ represents effective mobility, effective channel width, effective channel length, and $V_{\max }$ represents carrier maximum velocity.

As the drain bias is low, equation (13) can be written as $\mu_{\text {eff }} \cong \mu_{\mathrm{s}}$ and thus equation (12) can be rewritten as

$$
\beta_{\text {eff }}=\mu_{s} C_{o x} \frac{W_{\text {eff }}}{L_{\text {eff }}}
$$

It can be proved that, as $\theta\left(V_{\mathrm{GS}}-\mathrm{V}_{\mathrm{TH}}\right)^{2}=1$ in the mobility model proposed by Ghibaudo (equation (4)), the surface mobility $\mu_{s}$ will get its maximum value

$$
\mu_{\mathrm{s}}=\mu_{\mathrm{m}}\left(\mathrm{V}_{\mathrm{GS}}-\mathrm{V}_{\mathrm{TH}}\right)
$$

Take the derivative of $I_{D S}$ versus $V_{G s}$ in equation (11), we obtain the relationship

$$
\beta_{\mathrm{eff}}=\frac{1}{\mathrm{~V}_{\mathrm{DS}}} \frac{\mathrm{d} \mathrm{I}_{\mathrm{DS}}}{\mathrm{d} \mathrm{V}_{\mathrm{GS}}}
$$

Suppose we take derivative of $I_{D S}$ versus $V_{G S}$ and select the maximum value from the measured $I_{D S}$ versus $V_{G S}$ curve and divided this value by $V_{D S}$, then the following relationship exists

$$
\frac{1}{V_{D S}} \frac{d l_{D S}}{d V_{G S}}=\beta_{\text {eff }}(\max )=\mu_{s}(\max ) C_{o x} \frac{w_{\text {eff }}}{L_{\text {eff }}}=\mu_{m}\left(V_{G S}-V_{T H}\right) C_{o x} \frac{w_{e f f}}{L_{e f f}}
$$

By substitute the value of $V_{G S}$ as the $\mathrm{dI}_{D S} / \mathrm{dV}_{\mathrm{GS}}$ value is a maximum into equation (18), the fitting parameter $\mu_{m}$ can be extracted. At the same time, substitute this $V_{G S}$ into equation $\theta\left(V_{G S}-V_{T H}\right)=1$, the fitting parameter $\theta$ can also be extracted.

We believe that implementation of both the temperature dependence of threshold voltage (in section 3 ) and carrier mobility (in section 4) in SPICE MOS level 3, and with the mobility extraction described in the above section, the temperature dependence of drain current versus temperature can be predicted.

\section{Conclusion}

The temperature dependence of the threshold voltage can be predicted by theoretical prediction with quite good result. Two empirical surface mobility model are examined and better model is selected as SPICE level 3 model. Parameter extraction scheme for the new mobility model is proposed.

\section{Acknowledgments}

The authors are grateful to Mr. Ja-Hwai Ho and all the colleagues of semiconductor laboratory at Chung Cheng Institute of Technology for their helping in the experiments.

\section{References}

[1] Scribner D. A., Kruer M. R., and Killiany J. M., Proc. of the IEEE 79(1991)65-85.

[2] Massobrio G., and Antognetti P., Semiconductor Device Modeling (McGraw-Hill, Singapore, 1993) pp. 161-249.

[3] Wong H. S., White M. H., Krutsick T. J., and Booth R. V., SSE 30(1987)953. 
[4] Booth R. V., White M. H., Wong H. S., and Krutsick T. J., IEEE Trans. ED 34(1987)2501.

[5] Arora N. D., and Gildenblat G. Sh., IEEE Trans. ED 34(1987)89.

[6] Wang R., Dunkley J., DeMassa T. A., and Jelsma L. F., IEEE Trans. ED 18(1971)386.

[7] Wolf S., Silicon Processing for the VLSI era, (Lattice Press, California, 1990).

[8] Press W. H., Teukolsky S. A., Vetterling W. T., and Flannery B. P., Numerical Recipes in C, (Cambridge University Press, London, 1992).

[9] Emrani A., Balestra F., and Ghibaudo G., IEEE Trans. ED 40(1993)564.

[10] Yue C., Agostinelli Jr. V. M., Yeric G. M., Tasch Al F., IEEE Trans CAD of IC and Systems, 12(1993)1542. 


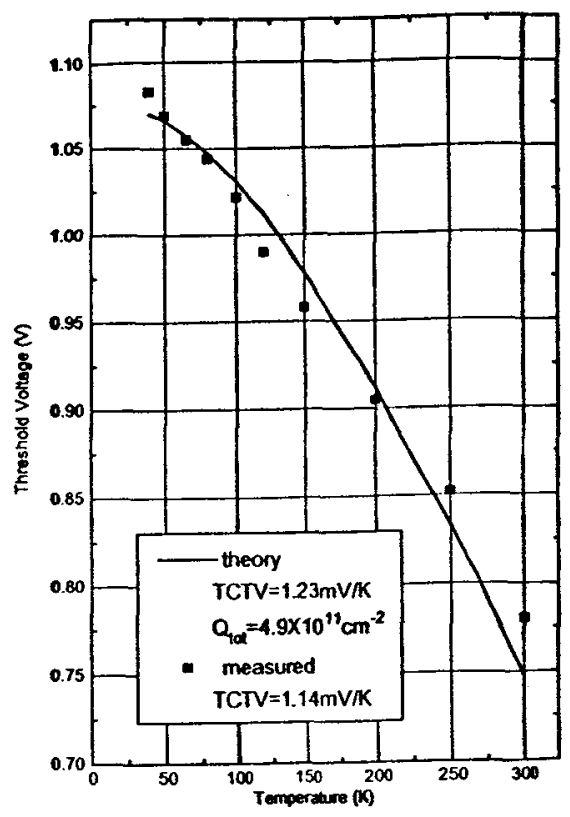

Figure 1. Temperature dependence of threshold vallage (NMOSFET $W / \mathrm{L}=50 / 50)$

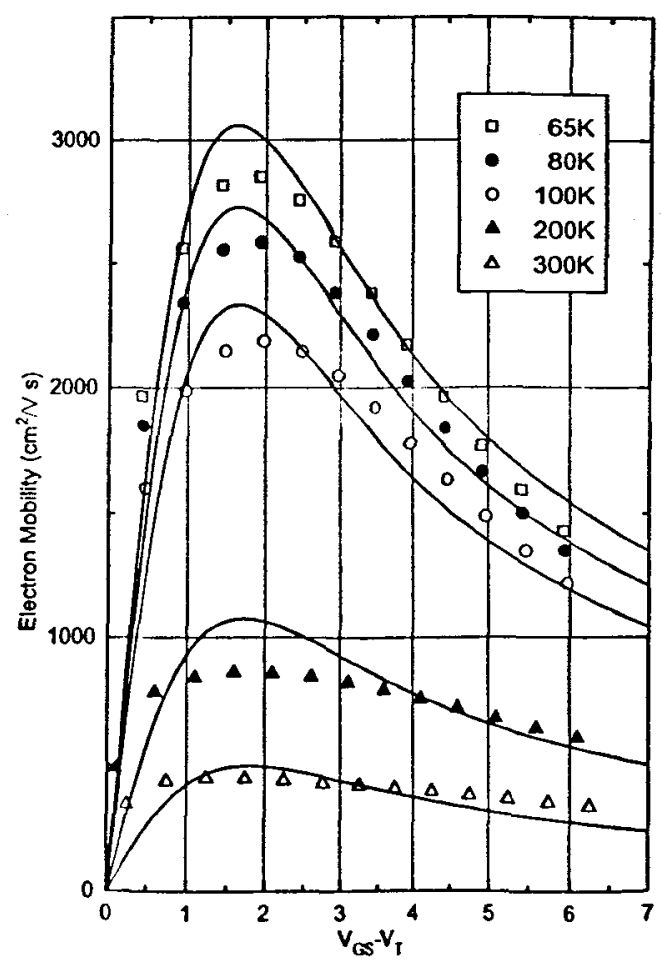

Figure 3. Ghibaudo's model (ine) compare with measured (dot) electron mobility

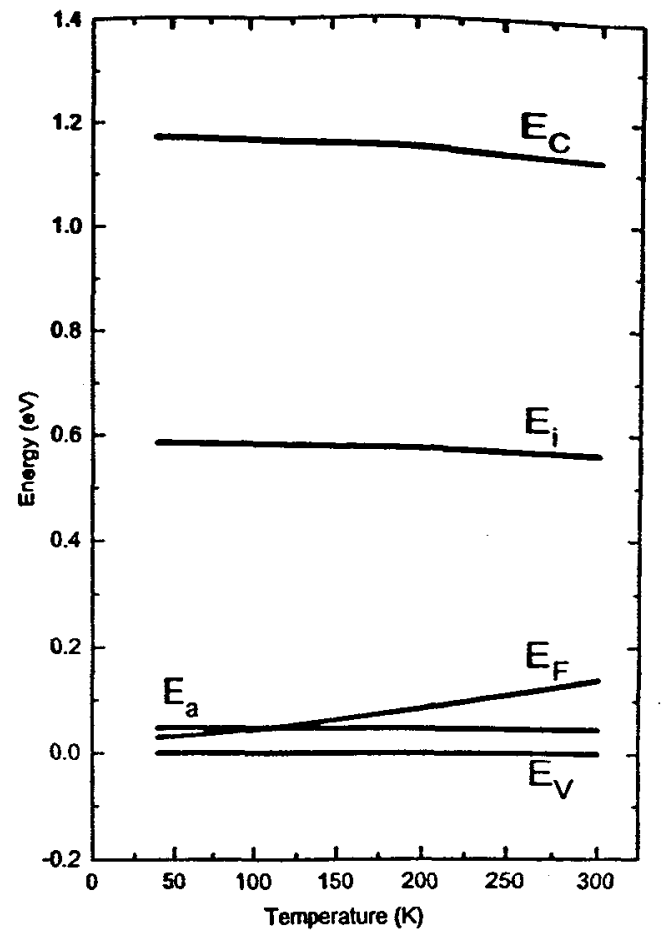

Figure 2. Band Diagram(all curves are plotted against fixed $\mathrm{E}_{\mathrm{v}}$ )



Figure 4. Temperature dependence of the fitting parameter of the Ghibaudo's model 




Figure 5. Tasch's model compare will measured electron mobility

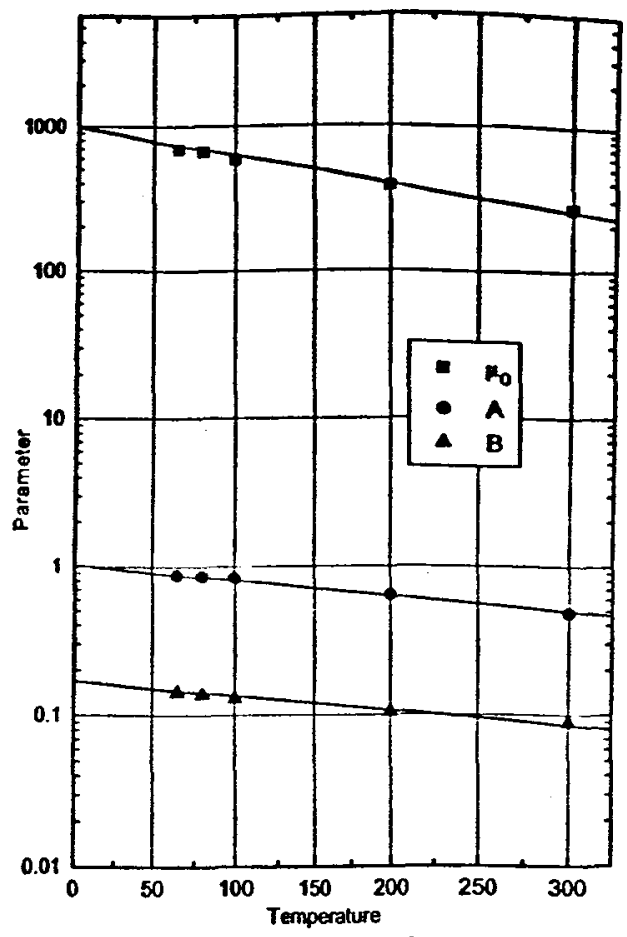

Figure 6. Temperature dependence of the fitting parameter of the Tasch's model 